Cite this: Phys. Chem. Chem. Phys., 2013,

\title{
Study of the tryptophan-terbium FRET pair coupled to silver nanoprisms for biosensing applications
}

15, 8838

Received 18th February 2013, Accepted 13th April 2013

DOI: $10.1039 / \mathrm{c} 3 \mathrm{cp} 50742 d$

www.rsc.org/pccp

\author{
Ane K. di Gennaro, ${ }^{a}$ Leonid Gurevich, ${ }^{a}$ Esben Skovsen, ${ }^{a}$ Michael T. Overgaard ${ }^{\mathrm{b}}$ and \\ Peter Fojan*a
}

\begin{abstract}
Plasmonic coupling between fluorophores and metal surfaces has become a focal point of optical research during the last two decades, however, the interactions of FRET couples with metal surfaces remain relatively unexplored. In this study, interactions of the tryptophan-Tb ${ }^{3+}$ FRET pair with silver nanoprisms for potential biosensor development have been investigated. For this purpose an engineered lanthanide binding peptide (LBTtrp) containing tryptophan as the sensitizer for bound lanthanide ions $\left(\mathrm{Tb}^{3+}\right)$ as well as a trypsin cleavage site was synthesized. The modified LBTtrp peptide contained two $\mathrm{N}$-terminal cysteine residues to provide a stronger coupling to the silver nanoprisms ( $\sim 6 \mathrm{~nm}$ high, $\sim 50 \mathrm{~nm}$ wide). This study investigated the interaction between tryptophan, chelated $\mathrm{Tb}^{3+}$ ions, and silver nanoprisms in solution using fluorescence and transient absorption spectroscopy. We have found that $\mathrm{Tb}^{3+}$ luminescence decreases upon binding of the LBTtrp- $\mathrm{Tb}^{3+}$ to silver nanoprisms and increases upon trypsin cleavage. The transient absorption spectroscopy measurements showed a significant decrease in the lifetime of the excited singlet state of tryptophan upon $\mathrm{Tb}^{3+}$ chelation, while coupling to the silver nanoprisms did not show a significant effect on tryptophan. The results obtained in this work demonstrate a first proof of concept for a new sensitive optical biosensor in solution.
\end{abstract}

\section{Introduction}

Nanomaterials science has evolved from dealing with pure physics and inorganic materials towards the development of advanced building blocks for biotechnological applications. Their unique properties have the potential to boost the development of new biosensors and to drastically broaden their application within environmental, industrial and biomedical sensing applications. This also includes medical diagnostics, proteomics and drug discovery. The increasing demand for enhanced sensitivity as well as improved selectivity of the biosensors spurs optimization and development of new types of biosensors. The phenomenon of localized plasmon resonance (LPR) in metallic nanoparticles has made them one of the main subjects of interest for the development of new biosensors. ${ }^{1,2}$ As a remarkable example, strong LPR scattering of gold nanoparticles coupled to fluorescently labeled biomolecules has improved the diagnostics of cancer as well as several other diseases. ${ }^{1}$ Recent developments

\footnotetext{
${ }^{a}$ Department of Physics and Nanotechnology, Aalborg University, Skjernvej 4A, 9220 Aalborg, Denmark. E-mail: fp@nano.aau.dk; Fax: +45 99409235; Tel: +4599407488

${ }^{b}$ Department of Biotechnology, Chemistry and Environmental Engineering, Aalborg University, Sohngaardsholmsvej 47, 9000 Aalborg, Denmark. E-mail: mto@bio.aau.dk; Tel: +4599408525
}

in nanoparticle synthesis technology allow for the efficient production of metal nanoparticles with tunable absorption spectra (colors). ${ }^{3}$ This can be achieved by varying the shape and dimensions of the metal nanoparticle ${ }^{4}$ or the metal shell in a core-shell particle ${ }^{5}$ and thus, changing the metal interaction with fluorophores on the surface. Thin metal surfaces made of gold or silver are also known to induce quenching of excited states via energy transfer to the metal surface, which is a phenomenon of considerable interest in biophotonics. ${ }^{3,6}$

For several years lanthanides have been used for protein labeling allowing for sensing and visualization of dynamical processes in proteins, localization of proteins in cells and tissues, as well as protein-protein interactions. ${ }^{7}$ For this purpose, peptide chelates for lanthanide ions have been designed and improved using protein engineering methods over the years. The chelate includes a lanthanide binding tag (LBT), comprising 15-20 amino acids and has to achieve several purposes: (1) high affinity and specific binding of the lanthanide ion, (2) provide the attachment of the lanthanide to a macromolecule, (3) prevent $\mathrm{H}_{2} \mathrm{O}$ coordination with the lanthanide, which otherwise would lead to luminescence quenching, and (4) contain a covalently coupled sensitizer molecule. The sensitizer molecule should exhibit a high absorption coefficient compared to the relatively small absorption cross section of lanthanides. ${ }^{8}$ A good sensitizer for such a system is 


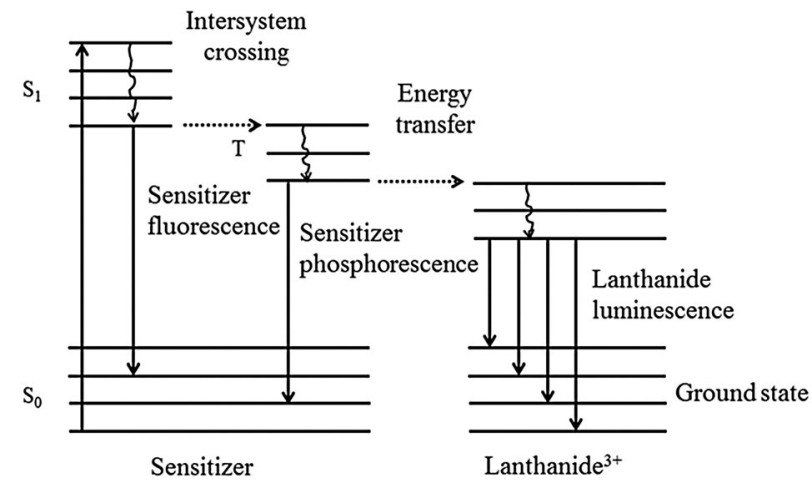

Fig. 1 Energy diagram of the lanthanide complex, consisting of a sensitizer and a lanthanide(III) ion. Upon sensitizer excitation, intersystem crossing to the sensitizer triplet state leads to energy transfer to the lanthanide(III) ion.

tryptophan (Trp), which has a molar extinction coefficient of $5800 \mathrm{~cm}^{-1} \mathrm{M}^{-1}$ at $280 \mathrm{~nm}$, while the molar extinction coefficient of $\mathrm{Tb}^{3+}$ is approximately $0.2 \mathrm{~cm}^{-1} \mathrm{M}^{-1}$ at the same excitation wavelength. ${ }^{9,10}$ Inserting such a sensitizer allows for lanthanide excitation using common fluorescence spectroscopy setups. ${ }^{8}$

Recent studies have shown that upon excitation of the sensitizer molecule, positioned in the LBT, the sensitizer transfers its energy to the lanthanide ion. This occurs via a three stage intersystem crossing mechanism involving transfer from the singlet excited state to the triplet state and then to the lanthanide ion (Fig. 1). ${ }^{11}$ The advantages of using lanthanides over molecular chromophores are: (1) long luminescence lifetimes, in the millisecond range, (2) narrow luminescence bands with large Stoke shifts (>200 nm), and (3) no bleaching. ${ }^{11,12}$ Long lifetimes offer the advantage of reducing the background fluorescence signal thus increasing the detection sensitivity. ${ }^{11}$

The photophysical and photochemical properties of Trp have been described in several reviews, discussing two different relaxation pathways from the singlet excited state of Trp $\left({ }^{1} \operatorname{Trp}^{*}\right)$. These two different pathways are described in eqn (1) and (2).

$$
\begin{gathered}
\operatorname{Trp}+h \nu \rightarrow{ }^{1} \operatorname{Trp}^{*} \rightarrow{ }^{1} \operatorname{Trp}{ }^{\bullet+}+\mathrm{e}^{-} \text {aq } \\
\operatorname{Trp}+h \nu \rightarrow{ }^{1} \operatorname{Trp}^{*} \rightarrow{ }^{3} \operatorname{Trp}
\end{gathered}
$$

The first relaxation pathway (eqn (1)) involves electron ejection from ${ }^{1} \mathrm{Trp}^{*}$ resulting in a positively charged Trp radical $\left({ }^{1} \operatorname{Trp}^{\bullet+}\right)$ and a solvated electron $\left(\mathrm{e}^{-}\right.$aq $)$. The second relaxation pathway (eqn (2)) involves intersystem crossing from the excited singlet state to the excited triplet state $\left({ }^{3} \operatorname{Trp}\right) .{ }^{13,14}$

Already a few decades ago, effects related to plasmonic coupling of fluorophores such as enhanced fluorescence on colloidal metal surfaces were observed. ${ }^{15}$ Due to the increasing demand for high sensitivity biomarker detection, this interaction has been investigated intensively using different metal nanostructures such as gold or silver nanoparticles ${ }^{4}$ or silver island films. ${ }^{16,17}$ The distance between the fluorophore and the metal surface determines whether the fluorescence is enhanced or quenched. In order to enhance the fluorescence signal, the optimal surface-fluorophore distance should be around $10 \mathrm{~nm}$, depending on the metal. ${ }^{18}$ When decreasing the distance, quenching by the metal dominates the interaction due to non-radiative energy transfer to the metal. ${ }^{17,18}$ On the other hand, the enhancement effect of the metal on the fluorescence emission decreases progressively with increasing separation distances between the fluorophore and the metal surface. ${ }^{15,19}$ In this respect, it can be expected that coupling and uncoupling of the tryptophan- $\mathrm{Tb}^{3+}$ complex from the metal surface would produce a significant effect on $\mathrm{Tb}^{3+}$ luminescence. To our knowledge the interaction of the Trp- $\mathrm{Tb}^{3+}$ FRET couple with a metal surface has not been reported before. The study of such an interaction could shed light on the energy transfer mechanism between ${ }^{1} \mathrm{Trp}^{*}$ and $\mathrm{Tb}^{3+}$ in the presence of silver nanoprisms (AgPr). From an applied science perspective, this effect could form the basis of an optical biosensor for real-time monitoring of proteolytic cleavage, capable of measuring the activity of medically relevant proteolytic enzymes. For example, it could be applied for measurements of the activity of ADAMTS-13 which is an important enzyme involved in hemostasis and for which a sufficiently selective and sensitive assay is currently not available. ${ }^{20,21}$ In this paper we investigated the effect of plasmonic coupling in a model system for a proteolytic cleavage assay, containing the tryptophan$\mathrm{Tb}^{3+}$ FRET pair and AgPr.

\section{Experimental}

\section{Protein solutions and chemicals}

Trypsin from Porcine pancreas was purchased from Sigma. Solutions of both trypsin and LBTtrp (synthesized in-house) were prepared by dissolving the protein powder in $10 \mathrm{mM}$ tris $\mathrm{HCl}$ buffer $\mathrm{pH}$ 7.5. Trypsin and LBTtrp concentrations were estimated using absorption spectroscopy measuring the absorbance at $280 \mathrm{~nm}$ and using the molar extinction coefficient of $37700 \mathrm{M}^{-1} \mathrm{~cm}^{-122}$ for trypsin and the computed molar extinction coefficient of $5625 \mathrm{M}^{-1} \mathrm{~cm}^{-1}$ by ProtParam ${ }^{23}$ for LBTtrp. Terbium(III) chloride hexahydrate was purchased from Strem Chemicals. Stock solution of terbium(III) chloride hexahydrate was prepared by dissolving the powder in deionized water. Tris $\mathrm{HCl}$ buffer was prepared using Trizma base from Sigma and $\mathrm{pH}$ adjusted by adding $5 \mathrm{M} \mathrm{HCl}$. Trisodium citrate, poly(sodium 4-styrene sulfonate), sodium borohydrate, and silver nitrate were purchased from Sigma. All chemicals were used as supplied without further purification.

\section{Preparation of silver prisms}

Silver nanoparticle seeds were synthesized by preparing a solution of $2.5 \mathrm{mM}$ trisodium citrate, $14 \mu \mathrm{M}$ poly(sodium 4-styrene sulfonate) and $60 \mu \mathrm{M}$ sodium borohydrate. To the freshly prepared solution $5 \mathrm{~mL} 500 \mu \mathrm{M}$ silver nitrate was added dropwise (approximately $1 \mathrm{~mL} \mathrm{~min}^{-1}$ ) with constant stirring at room temperature. The final silver nitrate and sodium borohydrate ratio was $1: 4$.

$500 \mu \mathrm{L}$ of the silver seed solution was slowly transferred into $6 \mathrm{~mL} 150 \mu \mathrm{M}$ ascorbic acid under constant stirring. The synthesis of AgPr was carried out by dispensing $3 \mathrm{~mL}$ freshly prepared $500 \mu \mathrm{M}$ silver nitrate from a buret $\left(0.1 \mathrm{~mL} \mathrm{~min}^{-1}\right)$ with 
constant stirring. 3 batches of AgPr were prepared and mixed after the synthesis. The final AgPr solution was characterized using UV-VIS spectroscopy, atomic force microscopy (AFM) and Nanosight Tracking Analysis (NTA).

\section{LBTtrp peptide synthesis}

LBTtrp (CCGAKSAFIDTNNDGWIEGDELLE) was synthesized in an automated solid-phase peptide synthesizer from Activotec (Activo P11) on rink amide MBHA resin (Advanced ChemTech) with Fmoc protected amino acids (Advanced ChemTech) producing amidated peptides. The peptide was cleaved from the resin by the addition of $3 \mathrm{~mL}$ cleavage solution (2.5\% TIS, 2.5\% EDT, 2.5\% Milli-Q water and $92.5 \%$ TFA). The cleavage reaction was carried out for 60 min under continuous shaking and the resin was washed with $3 \mathrm{~mL}$ TFA. The peptide was precipitated by the addition of $10 \mathrm{~mL}$ ice-cold diethylether. The sample was centrifuged for $10 \mathrm{~min}$ at $4500 \mathrm{rcf}$ and the supernatant decanted. The precipitate was freeze-dried, flushed with argon and stored at $-20{ }^{\circ} \mathrm{C}$.

\section{D Peptide structure modeling with Yasara and peptide sequence analysis with ProtParam}

The 3D structure of the synthesized LBTtrp peptide was predicted with the help of the modeling program Yasara. The final model of LBTtrp was energy minimized with the Amber force field in Yasara. The obtained 3D structure was displayed using RasWin molecular Graphics Windows Version 2.7.5.2. Distances between $\mathrm{Tb}^{3+}$ and the Trp residue, $\mathrm{Tb}^{3+}$ and the $\mathrm{SH}$-group of Cys2, and between Trp residue and the SH-group of Cys2 were estimated using the "monitor tool" in RasWin molecular Graphics Windows Version 2.7.5.2. ProtParam provided by ExPASy was used to predict physico-chemical parameters of the LBTtrp peptide sequence.

\section{Immobilization of LBTtrp onto AgPr and uncoupling by trypsin} cleavage

For each characterization technique, the following immobilization procedure was performed. A sample of $10 \mu \mathrm{M}$ LBTtrp in $10 \mathrm{mM}$ tris $\mathrm{HCl} \mathrm{pH} 7.5$ chelated with $196 \mu \mathrm{M} \mathrm{Tb}{ }^{3+}$ in the presence of $0.01 \mathrm{nM} \mathrm{AgPr}$ was incubated for 1.5 hours at room temperature under constant stirring. The same molar ratio between LBTtrp, $\mathrm{Tb}^{3+}$ and AgPr was used for each characterization technique. The sample was characterized immediately after incubation either using steady state fluorescence spectroscopy or using transient absorption spectroscopy.

Cleavage of the LBTtrp- $\mathrm{Tb}^{3+}$ peptide attached to the AgPr was conducted in the presence of $1: 40(\mathrm{w} / \mathrm{w})$ trypsin for 2 hours at room temperature under constant stirring. The sample was characterized immediately after incubation, either using steady state fluorescence spectroscopy or using transient absorption spectroscopy.

For localized plasmon resonance (LPR) detection using UV-VIS spectroscopy the uncoupled LBTtrp- $\mathrm{Tb}^{3+}$ as well as trypsin were removed from the solution by centrifugation at $4500 \mathrm{rcf}$ for $10 \mathrm{~min}$. After centrifugation the supernatant was decanted and the pellet was resuspended in buffer (initial volume).

\section{UV-VIS absorption spectroscopy}

A thermo scientific UV-VIS spectrophotometer (VWK International UV1 v4.60, West Chester, PA) was used to characterize synthesized AgPr, LBTtrp peptide, and peptide coated AgPr. The path length of the quartz cuvette was $1 \mathrm{~cm}$ and the acquired absorbance spectra were acquired in the range of 220-850 nm.

\section{AFM}

NT-MDT NTEGRA Aura multimode AFM was used to estimate the particle size and visualize the shape of the AgPr. The microscope was operated in intermittent contact mode using OMCL-AC160TS cantilevers (Olympus, $k=42 \mathrm{~N} \mathrm{~m}^{-1}, f=$ $300 \mathrm{kHz}$ ). A drop of AgPr solution was dried overnight on a mica wafer. The microscope was controlled using NTEGRA software Nova 1.0.26 RC1 and the captured images were plane subtracted in the program.

\section{Nanoparticle Tracking Analysis (NTA)}

NTA measurements were performed using a NanoSight LM12 (NanoSight, Salisbury, United Kingdom), equipped with a $640 \mathrm{~nm}$ laser. AgPr stock solution was diluted 10 times and injected into the sample chamber until the chamber was entirely filled without air bubbles. All measurements were performed at room temperature and the precise temperature monitored in the sample chamber. The software used for recording and analyzing the images was the NTA 2.1. The motion of AgPr in solution was recorded and tracked for $120 \mathrm{~s}$ using a manual shutter and gain adjustments. Three measurements of the AgPr batch were performed. The mean size and standard deviation (SD) were calculated using the NTA software based on the sizes of all the particles analyzed (total number of tracks $=17870$ ).

\section{Steady state fluorescence}

Fluorescence characterization of $10 \mu \mathrm{M}$ LBTtrp in the absence and the presence of $196 \mu \mathrm{M} \mathrm{Tb}^{3+}$ and $0.01 \mathrm{nM}$ AgPr was carried out using a RTC 2000 PTI spectrofluorimeter at room temperature. Also, fluorescence measurements of $\mathrm{Tb}^{3+}$ chelated LBTtrp coupled to AgPr were performed before and after trypsin cleavage of the peptide construct. The slit widths were set to $5 \mathrm{~nm}$ and the quartz cuvette path length was $1 \mathrm{~cm}$. Intrinsic LBTtrp fluorescence emission spectra and $\mathrm{Tb}^{3+}$ luminescence spectra were acquired with an excitation at $280 \mathrm{~nm}$. The spectra were acquired in the range of 320-450 and 570-610 $\mathrm{nm}$.

\section{Transient absorption}

The transient absorption studies were performed using a femtosecond laser system and a commercial transient absorption spectrometer (Helios, Ultrafast Systems LLC). The femtosecond oscillator was a mode locked Tsunami Ti:sapphire femtosecond laser (Spectra-Physics) pumped by a Millennia V diode laser. The $\sim 80$ fs pulses from the Tsunami were used to seed a chirped-pulse regenerative amplifier (Spectra-Physics, Spitfire, pumped by a high-power diode laser, Evolution, also from Spectra-Physics), yielding 100 fs pulses with $1 \mathrm{~mJ}$ of energy per pulse at a repetition rate of $1 \mathrm{kHz}$ (centered at $800 \mathrm{~nm}$ ). 
The output of the amplifier was divided into a high (95\%) and a low (5\%) power beam used to generate the pump and probe pulses, respectively. The probe beam was generated inside the Helios by white light continuum generation in a sapphire plate. The white light spectrum covered the range 420-700 nm. The $280 \mathrm{~nm}$ pump pulses were generated by frequency doubling the output of an optical parametric amplifier (Topas-White-SHS, Light Conversion). This resulted in a pump beam with an average power of $\sim 2 \mathrm{~mW}$ at a repetition rate of $1 \mathrm{kHz}$. The spectral width of the UV pump pulses was approximately $10 \mathrm{~nm}$ FWHM. The pump beam was passed through an optical delay line and a chopper at $500 \mathrm{~Hz}$ inside the Helios. Every second pump pulse was blocked by the chopper and the transient absorption with and without pump was recorded by the Helios. The transient absorption setup is depicted in Fig. 2.

The transient absorption spectra were measured for time delays between 545 ps and 3300 ps, and each sample was scanned 3 times and averaged. The transient absorption spectra were recorded using the software program supplied with the Helios absorption spectrometer.

The LBTtrp peptide concentration used for transient absorption experiments was $114 \mu \mathrm{M}$ in $10 \mathrm{mM}$ tris $\mathrm{HCl} \mathrm{pH} 7.5$ buffer. The sample was placed in a quartz cuvette with a path length of $0.1 \mathrm{~cm}$. Transient absorption spectra were acquired before and after the addition of $\mathrm{Tb}^{3+}$. Subsequently another set of transient absorption measurement was performed after AgPr addition. Transient absorption measurements were also acquired for the following solutions: (1) $114 \mu \mathrm{M}$ LBTtrp peptide with $0.07 \mathrm{nM}$ AgPr, (2) $10 \mathrm{mM}$ tris $\mathrm{HCl}$ pH 7.5, (3) $2.1 \mathrm{mM} \mathrm{Tb}^{3+}$ in buffer, (4) $0.07 \mathrm{nM}$ AgPr in buffer, (5) 114 $\mu \mathrm{M} \mathrm{Tb}{ }^{3+}$ with $0.07 \mathrm{nM}$ AgPr. The scans were carried out at $21 \pm 1{ }^{\circ} \mathrm{C}$ and the samples were continuously stirred in order to ensure homogeneous absorption of the biomolecules in solution.

\section{Data analysis}

Transient absorption spectroscopy. All spectra were smoothed using an adjacent averaging routine in MatLab (MathWorks).

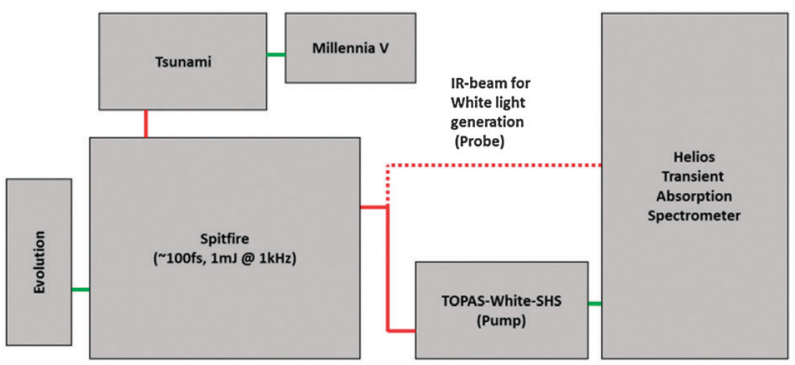

Fig. 2 A schematic of the transient absorption spectroscopy setup. The ultrafast amplified laser source consists of 6 components: a $532 \mathrm{~nm}$ continuous wave pump laser (Millenia V), a mode-locked titanium: sapphire laser (Tsunami), a $527 \mathrm{~nm}$ nanosecond pump laser (Evolution) and an amplification system (Spitfire). The majority of the power from the amplifier was used to generate $280 \mathrm{~nm}$ pump pulses inside an optical parametric amplifier (TOPAS-White-SHS), while a small part of the power was used for generation of broadband probe pulses by white light continuum generation in a sapphire plate inside the transient absorption spectrometer (Helios). The optical delay line inside the transient absorption spectrometer allowed the probe pulse to be delayed with respect to the pump pulse.
The spectrum of the buffer solution was subtracted from all transient absorption spectra before further analysis. These data sets were subsequently treated using Origin8 (OriginLab). Lifetime measurements of transient species at $560 \mathrm{~nm}$ were fitted with a single-exponential function (eqn (3)).

$$
y=A_{1} \times \exp \left(-x / \tau_{1}\right)+y_{o},
$$

where $y$ is the transient absorption at $560 \mathrm{~nm}, y_{0}$ is the transient absorption at zero time delay between the pump and the probe, $\tau_{1}$ is the lifetime and $A_{1}$ is the amplitude. All data were fitted according to a non-linear regression algorithm in Origin8.

Steady state fluorescence. The data were analyzed using Origin8. All spectra were buffer corrected by subtracting the spectrum of the buffer solution from the acquired sample spectra. Trp emission spectra were corrected for the dilution factor in the samples and normalized to their maximum fluorescence emission. The emission spectrum acquired from trypsin alone was subtracted from the Trp emission spectra and $\mathrm{Tb}^{3+}$ luminescence spectra after trypsin cleavage.

\section{Results and discussion}

\section{AgPr synthesis and characterization}

AgPr were synthesized by silver nitrate reduction as described in the Experimental section. A typical AFM image of AgPr showing the shape of the particles is depicted in Fig. 3.

The obtained particles were flat prisms with a predominant triangular shape and a typical size of $\sim 50 \mathrm{~nm}$ in width and $\sim 6 \mathrm{~nm}$ in height as observed using AFM. NTA revealed an average size of $33 \pm 1 \mathrm{~nm}$. The average size difference between AFM and NTA can be explained by the fact that the NTA program estimates a real time spherical particle size distribution in solution based on the Stokes-Einstein equation. The analyzed solution contained prism shaped nanoparticles, which would lead to a size under-estimation due to the non-spherical shape of

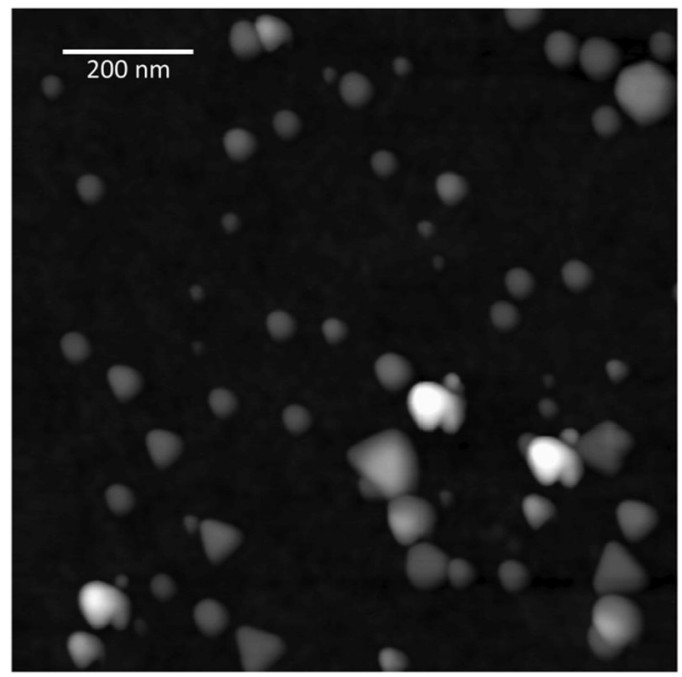

Fig. 3 AFM image of AgPr deposited onto a mica substrate. The estimated average size of the $\mathrm{AgPr}$ is $\sim 50 \mathrm{~nm}$ in width and $\sim 6 \mathrm{~nm}$ in height. 


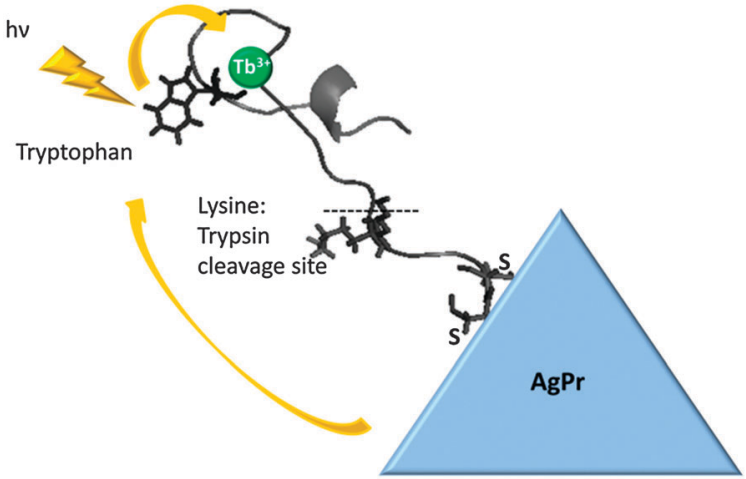

Fig. 4 Schematic of the biosensor model. AgPr is the silver nanoprism. The coupling between the AgPr and the LBTtrp peptide is achieved via two cysteine residues. The trypsin cleavage site is located after the lysine residue and 5 amino acids away from the $\mathrm{N}$-terminus of the peptide. $\mathrm{Tb}^{3+}$ is coordinated with the lanthanide binding tag consisting of 17 amino acids. The sensitizer, Trp, is depicted in ball and stick and $\mathrm{Tb}^{3+}$ as a sphere. The arrows indicate the studied interactions. The AgPr in the model is not drawn to scale.

the AgPr. Nanoparticle stock solution concentration was estimated using NTA to be approximately $3.4 \times 10^{14}$ particles per L.

\section{LBTtrp 3D structure and the biosensor model}

The physico-chemical properties of LBTtrp have been estimated by ProtParam on the basis of the fasta sequence: CCGAKSAFIDTNNDGWIEGDELLE revealing an isoelectric point of 3.71. The molecular weight of the peptide was calculated to be approximately $2600 \mathrm{~g} \mathrm{~mol}^{-1}$ while the extinction coefficient at $280 \mathrm{~nm}$ was estimated to be $5625 \mathrm{M}^{-1} \mathrm{~cm}^{-1}$. A schematic representation of the biosensor model system used to study the energy transfer between Trp, $\mathrm{Tb}^{3+}$ and AgPr is displayed in Fig. 4. The LBTtrp- $\mathrm{Tb}^{3+}$ structure in this figure is based on the modeled structure in Yasara.

Distances between $\mathrm{Tb}^{3+}$ and the Trp residue, $\mathrm{Tb}^{3+}$ and the SH-group of Cys2, and between Trp residue and the SH-group of Cys2 were measured using the Rasmol "monitor tool". Distances in the model were measured to be $7 \AA, 31 \AA$ and $33 \AA$ Å, respectively.

\section{Verification of LBTtrp coupling and uncoupling onto AgPr}

Synthesized AgPr were analyzed using UV-VIS absorption spectroscopy and revealed a single absorption band centered at $\sim 640 \mathrm{~nm}$ as shown in Fig. 5. The absorption peak can be assigned to the localized plasmon resonance of AgPr, which is in accordance with results found in the literature. ${ }^{24}$

Upon LBTtrp- $\mathrm{Tb}^{3+}$ coupling to the AgPr surface, via the engineered thiol/silver linker, a $45 \mathrm{~nm}$ red-shift was observed in the LPR absorption peak of AgPr. Trypsin cleavage of the peptide uncoupled the lanthanide binding moiety of the LBTtrp- $\mathrm{Tb}^{3+}$ peptide from AgPr resulting in a blue shift of the LPR absorption maximum of approximately $32 \mathrm{~nm}$. As can be observed in Fig. 5, the LPR absorption maximum of the AgPr decoupled from the LBTtrp peptide by trypsin displays a $13 \mathrm{~nm}$ red-shift indicating that the amino acids before the trypsin cleavage site in LBTtrp (CCGAK) remain attached to AgPr via

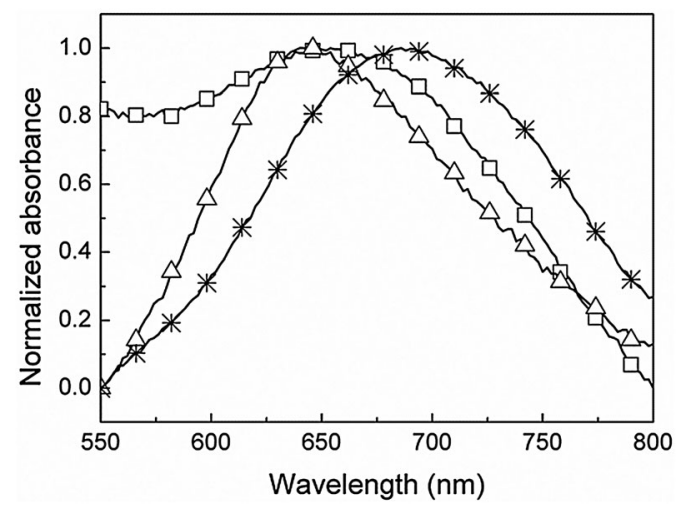

Fig. 5 Normalized absorption spectra of AgPr (triangles), LBTtrp-Tb ${ }^{3+}$ coupled to AgPr (stars) and LBTtrp-Tb ${ }^{3+}-\mathrm{AgPr}$ cleaved with trypsin (squares).

the two thiol groups. Moreover, LBTtrp binding to AgPr is preventing AgPr aggregation in solution upon $\mathrm{Tb}^{3+}$ addition (data not shown). These results are in accordance with the literature where several studies have confirmed the correlation between LPR absorption band shifts and changes in refractive index upon biomolecular coupling onto nanoparticle surfaces. ${ }^{25}$ In fact, several optical nanobiosensors based on LPR absorption band changes upon biomolecular coupling to the nanoparticle surface are already used in various fields such as drug design, clinical diagnostics and environmental control. ${ }^{2}$ The focus in this study is on the metal-Trp- $\mathrm{Tb}^{3+}$ interactions in the LBTtrp$\mathrm{Tb}^{3+}-\mathrm{AgPr}$ complex and its potential applications for biosensing.

\section{Metal-Trp-Tb ${ }^{3+}$ interactions in the LBTtrp- $\mathrm{Tb}^{3+}$-AgPr complex}

Fluorescence spectroscopy measurements were performed in order to investigate the effects of AgPr on the Trp fluorescence and $\mathrm{Tb}^{3+}$ luminescence upon LBTtrp- $\mathrm{Tb}^{3+}$ immobilization to AgPr. The measured $\mathrm{Tb}^{3+}$ luminescence spectra in the range coinciding with the AgPr LPR absorption peak are shown in Fig. 6.

Trp fluorescence of LBTtrp shows a noticeable decrease of $40 \%$ upon $\mathrm{Tb}^{3+}$ binding by LBTtrp, while $\mathrm{Tb}^{3+}$ luminescence at the same time increases (Fig. 6 and 7) confirming the sensitizing effect of Trp on $\mathrm{Tb}^{3+}$, as has been stated before. ${ }^{26}$

Immobilization of LBTtrp- $\mathrm{Tb}^{3+}$ on AgPr leads to a $9 \% \mathrm{~Tb}^{3+}$ luminescence decrease, while decoupling of the $\mathrm{Tb}^{3+}$ binding moiety by trypsin cleavage from the AgPr surface yields a $22 \%$ increase in $\mathrm{Tb}^{3+}$ luminescence (Fig. 6 and $8 \mathrm{~A}$ ). No significant effect on Trp fluorescence was observed upon LBTtrp-Tb ${ }^{3+}$ coupling to AgPr, whereas uncoupling by trypsin leads to a 17\% decrease in Trp fluorescence emission intensity (Fig. 7 and $8 \mathrm{~B})$. These data suggest that plasmonic coupling between $\mathrm{Tb}^{3+}$ and AgPr has a quenching effect on the $\mathrm{Tb}^{3+}$ excited state, while only a negligible quenching by AgPr was observed on the Trp excited state. In addition, upon uncoupling of LBTtrp-Tb ${ }^{3+}$ from the AgPr Trp, fluorescence decreased while $\mathrm{Tb}^{3+}$ luminescence increased (Fig. 6 and 7). This indicates that the energy transfer between Trp and $\mathrm{Tb}^{3+}$ was improved compared to free LBTtrp- $\mathrm{Tb}^{3+}$. Since the same increase in $\mathrm{Tb}^{3+}$ luminescence and the decrease in Trp fluorescence were observed when 


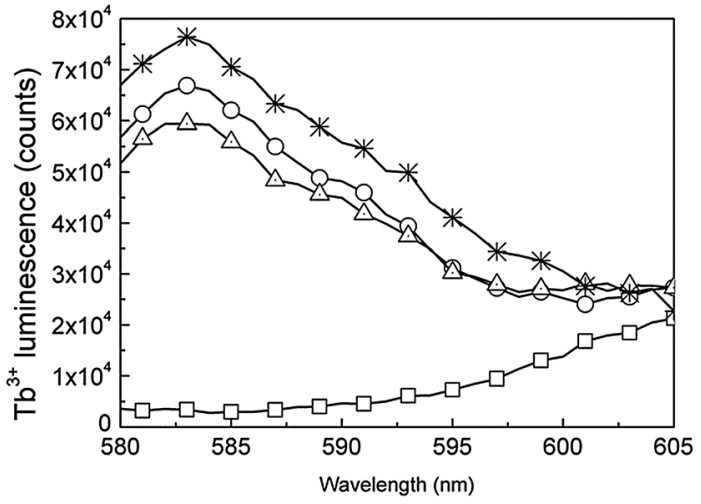

Fig. $6 \mathrm{~Tb}^{3+}$ luminescence upon sensitizer (Trp) excitation at $280 \mathrm{~nm}$. The spectra show $\mathrm{Tb}^{3+}$ luminescence of free LBTtrp (squares), LBTtrp chelated with $\mathrm{Tb}^{3+}$ (circles), LBTtrp-Tb ${ }^{3+}$ coupled to AgPr (triangles) and LBTtrp-Tb ${ }^{3+}$ uncoupled from AgPr by trypsin cleavage of the LBTtrp peptide (stars).

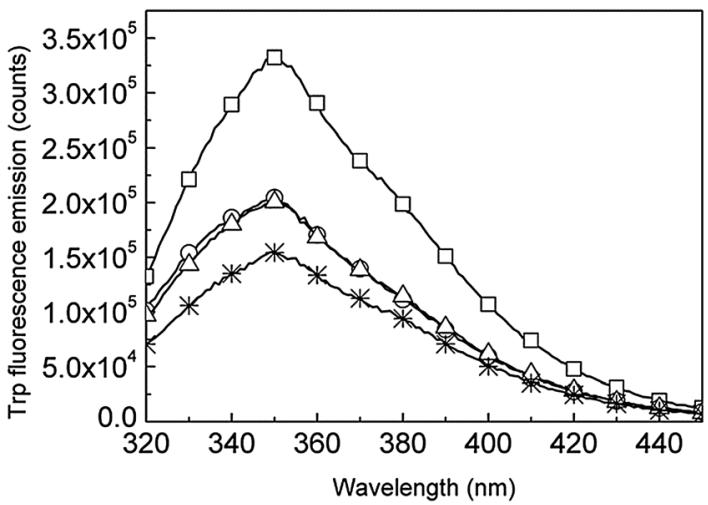

Fig. 7 Trp fluorescence emission spectra upon $280 \mathrm{~nm}$ excitation of free LBTtrp (squares), $\mathrm{Tb}^{3+}$-bound LBTtrp (circles), LBTtrp-Th ${ }^{3+}$ immobilized onto AgPr (triangles) and LBTtrp-Th ${ }^{3+}$ uncoupled from AgPr by trypsin cleavage of the peptide (stars).
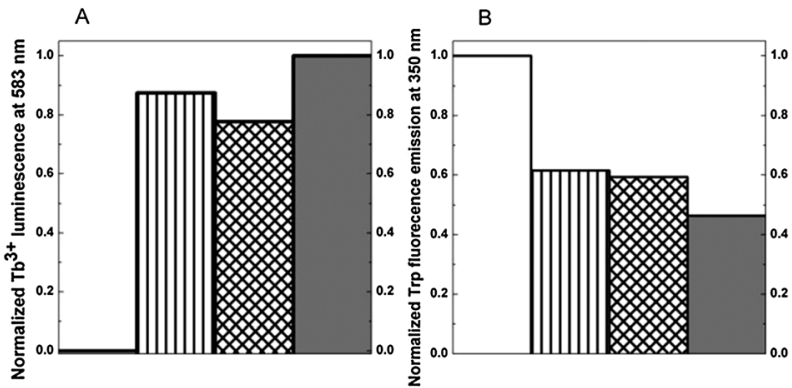

Fig. 8 Normalized $\mathrm{Tb}^{3+}$ luminescence (A) and normalized Trp fluorescence emission (B) upon excitation at $280 \mathrm{~nm}$ of free LBTtrp (blank), Tb ${ }^{3+}$-bound LBTtrp (vertically hatched), LBTtrp-Tb ${ }^{3+}$ immobilized on AgPr (cross hatched) and LBTtrp-Tb ${ }^{3+}$ uncoupled from AgPr by trypsin cleavage of the peptide (grey).

cleaving free LBTtrp- $\mathrm{Tb}^{3+}$ (data not shown), the observed change is most likely related to conformational rearrangements in the LBTtrp peptide upon cleavage.

In LBTtrp- $\mathrm{Tb}^{3+}$, Trp is positioned approximately $7 \AA$ away from the chelated $\mathrm{Tb}^{3+}$ ion, and thus enables intermolecular energy transfer to $\mathrm{Tb}^{3+}$, giving rise to $\mathrm{Tb}^{3+}$ luminescence.
As already mentioned, several scientific papers have been published on the energy transfer between lanthanides and various types of sensitizers. ${ }^{27}$ For a range of different sensitizers the energy transfer process between the sensitizer and the lanthanide ion has been described as energy transfer via the triplet excited state of the sensitizer to the chelated lanthanide ion, ${ }^{26}$ as depicted in Fig. 1 .

In order to study the behavior of the singlet Trp excited state $\left({ }^{1} \operatorname{Trp}^{*}\right)$ in the presence of chelated $\mathrm{Tb}^{3+}$ and AgPr, transient absorption measurements were performed. The results are summarized in Table 1.

The transient absorption spectra of free LBTtrp displayed a distinct transient absorption peak at $560 \mathrm{~nm}$ with a lifetime of 99 ps. Both the lifetime and the position of the absorption maximum are in agreement with photolytic studies of ${ }^{1} \mathrm{Trp}^{*}$ found in the literature. The particular transient absorption peak at $560 \mathrm{~nm}$, with a lifetime of approximately $400 \mathrm{ps}$, has previously been assigned to the transient absorption of aqueous ${ }^{1} \operatorname{Trp}^{*} .{ }^{13,14}$ This study focuses on ${ }^{1} \mathrm{Trp}^{*}$ in protic environments and therefore the transient absorption lifetime of ${ }^{1} \mathrm{Trp}^{*}$ is expected to be shorter. ${ }^{28}$ As depicted in Table $1, \mathrm{~Tb}^{3+}$ binding to the LBTtrp peptide leads to a significant decrease in ${ }^{1} \mathrm{Trp}^{*}$ lifetime, but coupling of LBTtrp to AgPr does not, within the accuracy of the measurements, displays any further decrease in lifetime. These results are consistent with the observation that the immobilization of LBTtrp to AgPr in the absence of $\mathrm{Tb}^{3+}$ causes no significant change in ${ }^{1} \mathrm{Trp}^{*}$ lifetime. Regarding the energy transfer between Trp and $\mathrm{Tb}^{3+}$, the observed decrease in ${ }^{1} \mathrm{Trp}^{*}$ lifetime upon $\mathrm{Tb}^{3+}$ binding to LBTtrp can be interpreted in several ways: (1) increased nonradiative decay of ${ }^{1} \mathrm{Trp}^{*}$, (2) increased direct energy transfer between ${ }^{1} \mathrm{Trp}^{*}$ and $\mathrm{Tb}^{3+}$, (3) the increased rate of intersystem crossing between ${ }^{1} \mathrm{Trp}^{*}$ and ${ }^{3} \mathrm{Trp}$, which could indirectly increase energy transfer to $\mathrm{Tb}^{3+}$ from the ${ }^{3} \mathrm{Trp}$. If the non-radiative decay rate of ${ }^{1} \mathrm{Trp}^{*}$ increases this event should suppress photo sensitisation of $\mathrm{Tb}^{3+}$. However, efficient photo sensitisation of $\mathrm{Tb}^{3+}$ was indeed observed. In principle, both the second and the third possibility agree with the achieved data. If the energy transfer occurs between ${ }^{3} \operatorname{Trp}$ and $\mathrm{Tb}^{3+}$, the lifetime of ${ }^{3} \operatorname{Trp}$ should decrease upon $\mathrm{Tb}^{3+}$ binding. A second transient absorption peak was observed at $\sim 450 \mathrm{~nm}$, which has previously been assigned to ${ }^{3} \mathrm{Trp} .{ }^{13} \mathrm{~A}$ minor difference in ${ }^{3} \operatorname{Trp}$ transient absorption was indeed observed between LBTtrp with and without $\mathrm{Tb}^{3+}$, but the signal to noise ratio at this wavelength was too low to determine whether the lifetime of ${ }^{3} \operatorname{Trp}$ was significantly decreased. In summary, the lifetime of ${ }^{1} \mathrm{Trp}^{*}$ is influenced by $\mathrm{Tb}^{3+}$ chelation

Table 1 Lifetimes of ${ }^{1} \operatorname{Trp}^{*}$ transient absorption at $560 \mathrm{~nm}$ upon $280 \mathrm{~nm}$ excitation of free LBTtrp, LBTtrp chelated with $\mathrm{Tb}^{3+}, \mathrm{LBTtrp}^{\mathrm{T} \mathrm{Tb}^{3+}}$ coupled to $\mathrm{AgPr}$, and LBTtrp coupled to AgPr in the absence of $\mathrm{Tb}^{3+}$. The uncertainty listed for the lifetimes is the standard error from the fitting routine

\begin{tabular}{|c|c|c|}
\hline & ${ }^{1} \operatorname{Trp}^{*} @ 560 \mathrm{~nm}(\tau / \mathrm{ps})$ & $R^{2}$ \\
\hline LBTtrp & $99 \pm 9$ & 0.97 \\
\hline LBTtrp $\mathrm{Tb}^{3+}$ & $37 \pm 6$ & 0.86 \\
\hline LBTtrp $\mathrm{Tb}^{3+} \mathrm{Ag} P \mathrm{Pr}$ & $28 \pm 4$ & 0.91 \\
\hline LBTtrp AgPr & $111 \pm 7$ & 0.98 \\
\hline
\end{tabular}


but no significant quenching of the ${ }^{1} \operatorname{Trp}^{*}$ by the AgPr was observed. These results support the interpretation of the steady state fluorescence studies that energy transfer occurs between Trp and $\mathrm{Tb}^{3+}$ and that the AgPr quenches $\mathrm{Tb}^{3+}$ luminescence but not Trp fluorescence emission.

\section{Conclusions}

The presented study demonstrates a responsive system based on AgPr and an engineered lanthanide binding peptide containing chelated $\mathrm{Tb}^{3+}$ with $\operatorname{Trp}$ as the sensitizer. The energy transfer between $\operatorname{Trp}$ and $\mathrm{Tb}^{3+}$ has been investigated using fluorescence spectroscopy and transient absorption spectroscopy. Coupling of LBTtrp- $\mathrm{Tb}^{3+}$ to AgPr was confirmed by an induced red-shift in the LPR absorption band. The detachment of the lanthanide binding moiety of the peptide from AgPr by tryptic digest was verified by a blue-shift of the LPR absorption band. When LBTtrp- $\mathrm{Tb}^{3+}$ was coupled to AgPr a 9\% decrease in $\mathrm{Tb}^{3+}$ luminescence was observed due to quenching. Subsequent decoupling of the lanthanide moiety from AgPr by trypsin increased the $\mathrm{Tb}^{3+}$ luminescence by $22 \%$.

This demonstrates the potential for applying the investigated system as a sensitive optical biosensor for enzymatic activity detection, as well as a sensitive diagnostic system for changes in enzymatic activities. The presented system is a demonstration of a plasmonic based biosensor in solution.

\section{Notes and references}

1 P. K. Jain, X. Huang, I. H. El-Sayed and M. A. El-Sayed, Acc. Chem. Res., 2008, 41, 1578.

2 B. Sepúlveda, P. C. Angelomé, L. M. Lechuga and L. M. Liz-Marzán, Nano Today, 2009, 4, 244.

3 P. Baptista, E. Pereira, P. Eaton, G. Doria, A. Miranda, I. Gomes, P. Quaresma and R. Franco, Anal. Bioanal. Chem., 2008, 391, 943.

4 A. J. Haes, S. Zou, G. C. Schatz and R. P. Van Duyne, J. Phys. Chem. B, 2004, 108, 109.

5 J. L. Lyon, D. A. Fleming, M. B. Stone, P. Schiffer and M. E. Williams, Nano Lett., 2004, 4, 719.
6 H. Imahori and S. Fukuzumi, Adv. Biomater., 2001, 13, 1197.

7 N. Soh, Sensors, 2008, 8, 1004.

8 P. R. Selvin, IEEE J. Sel. Top. Quantum Electron., 1996, 2, 1077.

9 H. Edelhoch, Biochemistry, 1967, 6, 1948.

10 E. I. Onstott and C. J. Brown, Anal. Chem., 1958, 30, 172.

11 S. Pandya, J. Yu and D. Parker, Dalton Trans., 2006, 2757.

12 B. R. Sculimbrene and B. Imperiali, J. Am. Chem. Soc., 2006, 128, 7346.

13 D. Creed, Photochem. Photobiol., 2008, 39, 537-562.

14 H. Kandori, R. F. Borkman and K. Yoshihara, J. Phys. Chem., 1993, 97, 9664-9667.

15 J. R. Lakowicz, Anal. Biochem., 2005, 337, 171.

16 E. Matveeva, Z. Gryczynski, J. Malicka, I. Gryczynski and J. R. Lakowicz, Anal. Biochem., 2004, 334, 303.

17 I. Gryczynski, E. G. Matveeva, P. Sarkar, S. Bharill, J. Borejdo, W. Mandecki, I. Akopova and Z. Gryczynski, Chem. Phys. Lett., 2008, 462, 327.

18 N. Akbay, J. R. Lakowicz and K. Ray, J. Phys. Chem. C, 2012, 116, 10766.

19 K. Ray, R. Badugu and J. R. Lakowicz, Langmuir, 2006, 22, 8374.

20 F. Peyvandi, R. Palla, L. A. Lotta, I. Mackie, M. A. Scully and S. J. Machin, J. Thromb. Haemostasis, 2010, 8, 631.

21 J. D. Studt, M. Bohm, U. Budde, J. P. Girma, K. Varadi and B. Lammle, J. Thromb. Haemostasis, 2003, 1, 1882.

22 C. N. Pace, F. Vajdos, L. Fee, G. Grimsley and T. Gray, Protein Sci., 1995, 4, 2411.

23 E. Gasteiger, C. Hoogland, A. Gattiker, S. Duvaud, M. R. Wilkins, R. D. Appel and A. Bairoch, The Proteomics Protocols Handbook, Humana Press Inc., 2005, pp. 571-607.

24 A. J. Frank, N. Cathcart, K. E. Maly and V. Kitaev, J. Chem. Educ., 2010, 87, 1098.

25 P. Englebienne, A. Van Hoonacker and M. Verhas, Spectroscopy, 2003, 17, 255.

26 M. H. V. Werts, J. W. Verhoevena and J. W. Hofstraat, J. Chem. Soc., Perkin Trans. 2, 2000, 433.

27 H. E. Rajapakse, D. R. Reddy, S. Mohandessi, N. G. Butlin and L. W. Miller, Angew. Chem., Int. Ed., 2009, 48, 4990.

28 Y. Chen, B. Liu, H. T. Yu and M. D. Barkley, J. Am. Chem. Soc., 1996, 118, 9271. 